\title{
Therapy-Resistant, Spontaneously Remitting Generalized Neutrophilic Eccrine Hidradenitis in a Healthy Patient Decreases the Expression of Dermcidin in Affected Eccrine Glands
}

\author{
Yumi Kambayashi Taku Fujimura Kenshi Yamasaki \\ Setsuya Aiba \\ Department of Dermatology, Tohoku University Graduate School of Medicine, Sendai, \\ Japan
}

\section{Key Words}

Neutrophilic eccrine hidradenitis · Dermcidin · Eccrine glands

\begin{abstract}
We describe a healthy 69-year-old Japanese man with generalized neutrophilic eccrine hidradenitis (NEH). He visited our outpatient clinic with a 15-year history of disseminated pruritic papules on his trunk and extremities; the eruptions, however, were limited to the summer months. Histological findings reveal a dense accumulation of neutrophils around the sweat glands with degenerated secretary coils. Interestingly, immunohistochemical staining showed that the expression of dermcidin on the secretory portion of eccrine glands was significantly decreased in the affected lesion. To our knowledge, this is the first report in English of generalized NEH in a healthy adult that shows the downregulation of the expression of dermcidin in affected eccrine glands.
\end{abstract}

\section{Introduction}

Neutrophilic eccrine hidradenitis (NEH) is known as an inflammatory disease of the eccrine sweat glands and is histologically characterized by necrosis and vacuolar degeneration of eccrine coil cells and dense neutrophil accumulation around the secretory coils [1]. Previous reports suggest that NEH normally develops in patients with malignancies following chemotherapy such as cytarabine, although hemodialysis, Behçet's disease or infectious diseases, or nonchemotherapeutic drugs such as 
granulocyte colony-stimulating factor (G-CSF) are also associated with NEH $[2,3]$. However, generalized NEH in healthy adults without any disorders is extremely rare and its precise pathogenesis is still under consideration.

Dermcidin is a dominant sweat antimicrobial peptide with broad-spectrum activity which is specifically and constitutively expressed in the sweat glands [4]. Minami et al. [5] reported that, in normal skin, the secretory coils of the eccrine sweat glands were positive for dermcidin and that, in contrast, the eccrine ducts lacked expression of dermcidin. This peptide is reported to be secreted into eccrine sweat directly. Therefore, to investigate the destruction of secretory coils of eccrine glands in NEH, in this report, we demonstrate immunohistochemical staining for dermcidin and give a possible explanation for the downregulation of dermcidin in secretory coils of affected eccrine glands in a patient with NEH.

\section{Case Report}

A 69-year-old Japanese man visited our outpatient clinic with a 15-year history of disseminated pruritic papules on his trunk and extremities; however, the eruptions were limited to the summer months. He had been treated with topical steroids and antihistamine without any improvement. Interestingly, the pruritic papules disappeared without any treatment after the outside temperature went below $20^{\circ} \mathrm{C}$ in fall. Physical examination showed numerous erythematous papules and plaques on the forearms and the back (fig. 1). A full blood count and biochemical profile were within normal range. He had a negative phototest for both UVA and UVB. The histopathological studies of the lesional skin revealed degeneration of the eccrine coils associated with dense accumulation of neutrophils (fig. 2). Moreover, immunohistochemical staining demonstrated that the secretory portion of the eccrine glands in the affected lesion significantly decreased the reactivity with antidermcidin antibody, while that in the nonaffected lesion was strongly stained with the antibody (fig. $3 a, b)$. As previously reported, the expression of dermcidin was not recognized in eccrine ducts [5]. Taking these clinical, histological, and immunohistological findings into account, we diagnosed this patient with generalized NEH. Without any additional treatment, the pruritic papules disappeared in the middle of September.

\section{Discussion}

In this report, we present a case of therapy-resistant generalized NEH in an otherwise healthy adult. Any treatment, including topical very strong or even the strongest steroid ointments and systemic antihistamines, was not effective. Of particular interest is that the eruptions spontaneously resolved in fall when the outside temperature went below $20^{\circ} \mathrm{C}$.

$\mathrm{NEH}$ is known as an inflammatory disease of the eccrine sweat glands. It is reported that NEH normally develops in patients with malignancies or following chemotherapy [1]. Actually, Bachmeyer and Aractingi [2] suggested that $90 \%$ of the NEH cases occur in patients with acute myeloid leukemia. Other associations include nonchemotherapeutic drugs such as granulocyte G-CSF, hemodialysis, Behçet's disease and infectious diseases (e.g. Serratia marcescens, Enterobacter cloacae, Staphylococcus aureus, streptococcal endocarditis, Nocardia, HIV-1) [2, 3, 6-9]. However, generalized NEH without any disorder is extremely rare. In fact, there is no case of generalized NEH in healthy adults in English literature. In healthy children, NEH has been reported as idiopathic palmoplantar hidradenitis $[10,11]$. Although 61 cases of idiopathic 
palmoplantar hidradenitis have been reported previously [10-12], the eruptions usually occur mainly on the soles and palms, and only 4 cases of generalized NEH in healthy children have been reported [11]. One possible trigger for the outbreak of generalized NEH is constantly high temperature. To sum up, our present case is the first report in English that describes generalized NEH in a healthy adult (table 1).

Although the pathogenesis of NEH is unclear, two major hypotheses have been proposed. The first one postulates that NEH is the consequence of a direct cytotoxicity of a drug secreted in the sweat in eccrine coils and duct cells, while the other one suggests that NEH is one from the spectrum of neutrophilic dermatoses, as are Sweet's syndrome and hematological malignancies [2]. Association with temporal intense physical activity and exposure to cold has been reported [13]. The onset is often associated with fever [14].

Dermcidin is constitutively secreted by the eccrine sweat glands and not induced by skin inflammation or injury [15]. It appears to be the dominant sweat antimicrobial peptide with broad-spectrum activity not affected by low $\mathrm{pH}$ or increased salt concentrations, and is known to be secreted into eccrine sweat directly. In our case, interestingly, the staining for dermcidin in affected secretory coils of eccrine glands, which were surrounded by significant numbers of neutrophils, were almost negative. In contrast, secretory portions of eccrine ducts without neutrophil accumulation expressed dermcidin and the staining level of dermcidin in the area not affected by NEH was almost similar to those in healthy adults. These results suggest that, in NEH, the secretory coils of eccrine glands may be destroyed by neutrophils, resulting in the decrease of sweat in the affected skin, and this may cause the spontaneous regression of the disorder.

In this report, we present a case of generalized NEH in a healthy adult. The precise mechanisms of NEH are still unknown. Our present case gives a possible explanation for the mechanism of NEH in a healthy adult but further evidence from more case reports is needed.

Table 1. Generalized neutrophilic eccrine hidradenitis in healthy patients

\begin{tabular}{lcll}
\hline $\begin{array}{l}\text { Case No. } \\
\text { [Reference] }\end{array}$ & Age of onset & $\begin{array}{l}\text { Distribution of } \\
\text { lesions }\end{array}$ & Possible reasons \\
\hline $1[18]$ & 7 months & Limbs, trunk & High temperature \\
$2[18]$ & 7 months & Limbs, trunk & High temperature \\
$3[17]$ & 14 months & Limbs, trunk & High temperature, excessive sweating \\
$4[17]$ & 10 months & Limbs, trunk & High temperature, excessive sweating \\
Present case & 69 years & Limbs, trunk & High temperature \\
\hline
\end{tabular}




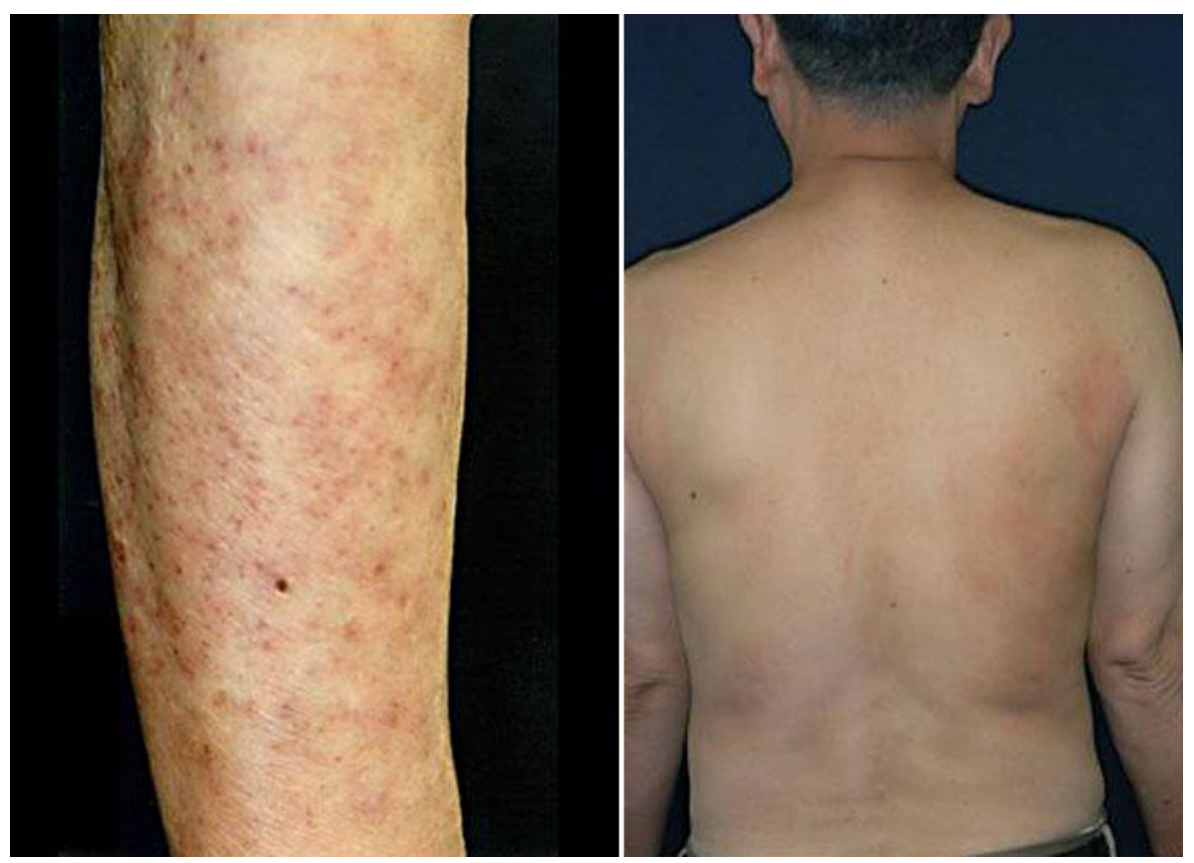

Fig. 1. Erythematous papules and plaques on the forearms. 


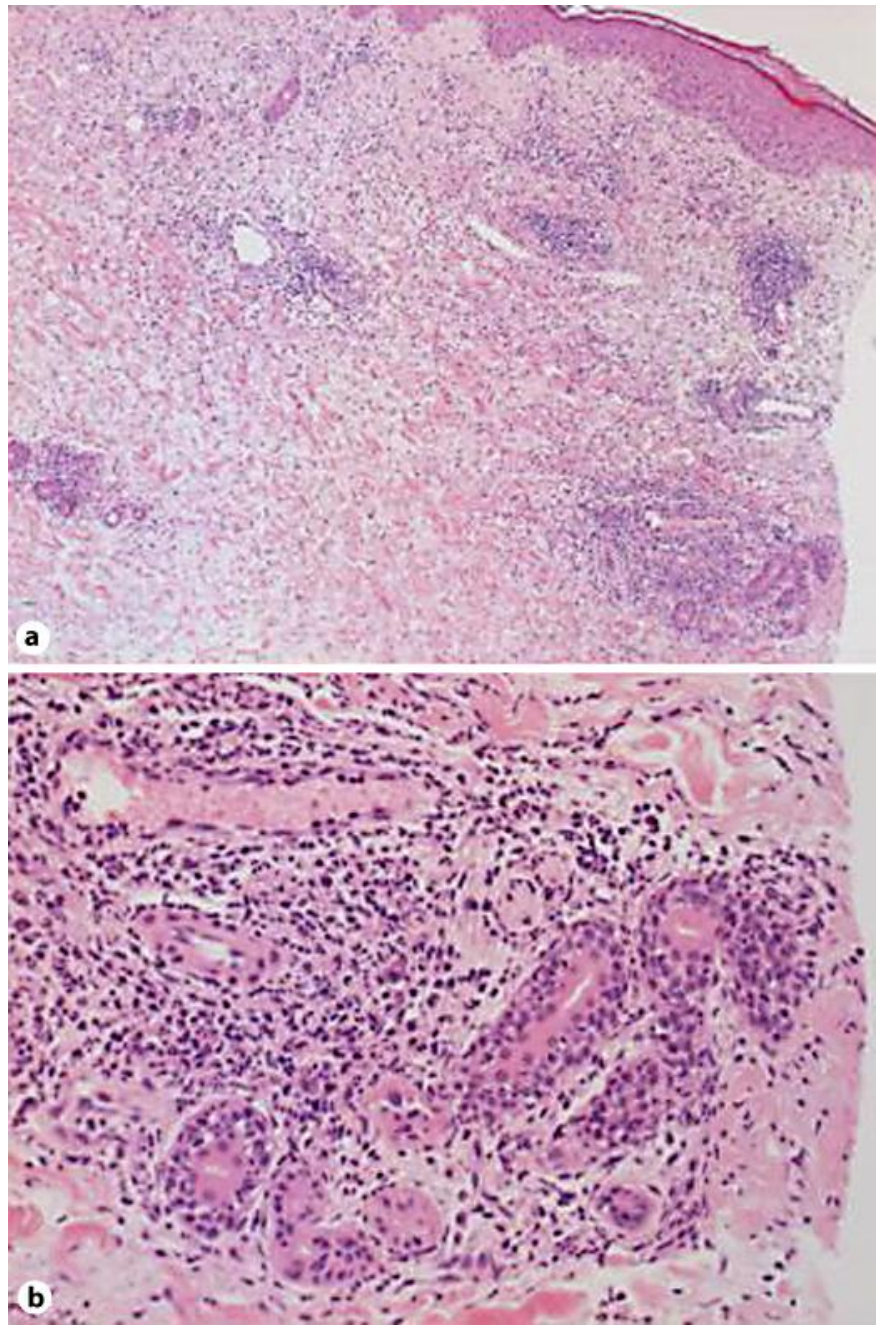

Fig. 2. a Dense accumulation of neutrophils in the deep dermis. b Degeneration of eccrine coils associated with densely accumulated neutrophils (original magnification $\mathbf{a} \times 50, \mathbf{b} \times 200$ ). 

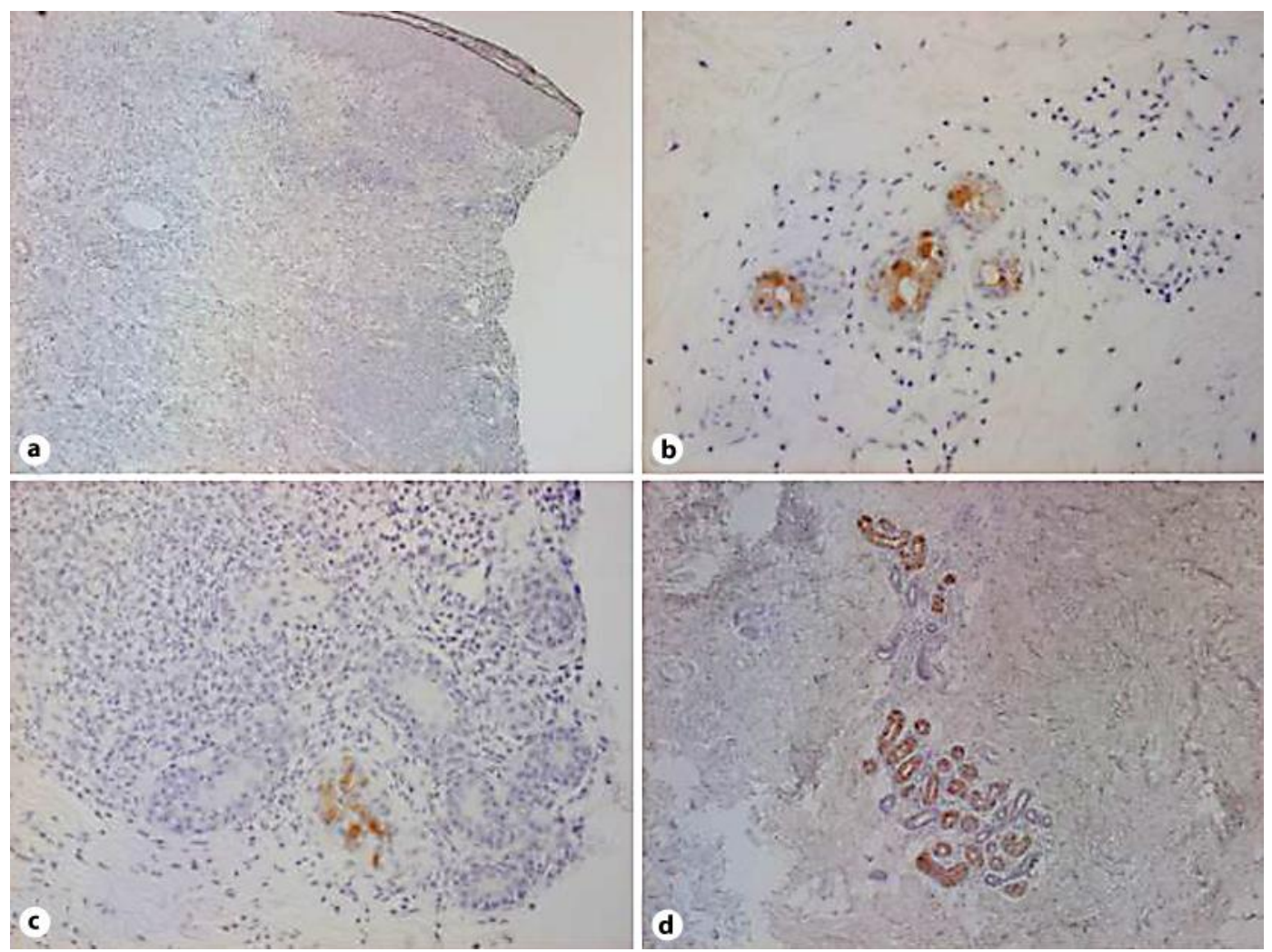

Fig. 3. The secretory portion of eccrine glands in the affected lesion significantly decreased the expression of dermcidin (original magnification $\mathbf{a} \times 50, \mathbf{b} \times 200$ ). The secretory portion of eccrine glands in a nonaffected lesion is strongly positive for dermcidin $(c \times 200)$. Secretory portion of eccrine glands in a healthy donor $(\mathbf{d} \times 200)$.

\section{References}

1 Harrist TJ, Fine JD, Berman RS, Murphy GF, Mihm MC Jr: Neutrophilic eccrine hidradenitis. A distinctive type of neutrophilic dermatosis associated with myelogenous leukemia and chemotherapy. Arch Dermatol 1982;118:263-266.

$\checkmark 2$ Bachmeyer C, Aractingi S: Neutrophilic eccrine hidradenitis. Clin Dermatol 2000;18:319-330.

-3 Nijsten TE, Meuleman L, Lambert J: Chronic pruritic neutrophilic eccrine hidradenitis in a patient with Behcet's disease. Br J Dermatol 2002;147:797-800.

-4 Wiesner J, Vilcinskas A: Antimicrobial peptides: the ancient arm of the human immune system. Virulence 2010;1:440-464.

5 Minami Y, Uede K, Sagawa K, Kimura A, Tsuji T, Furukawa F: Immunohistochemical staining of cutaneous tumours with G-81, a monoclonal antibody to dermcidin. Br J Dermatol 2004;151:165-169.

-6 Smith KJ, Skelton HG 3rd, James WD, Holland TT, Lupton GP, Angritt P: Neutrophilic eccrine hidradenitis in HIV-infected patients. Armed Forces Retrovirus Research Group. J Am Acad Dermatol 1990;23(5 Pt 1):945-947.

7 Combemale P, Faisant M, Azoulay-Petit C, Dupin M, Kanitakis J: Neutrophilic eccrine hidradenitis secondary to infection with Serratia marcescens. Br J Dermatol 2000;142:784-788.

8 Antonovich DD, Berke A, Grant-Kels JM, Fung M: Infectious eccrine hidradenitis caused by Nocardia. J Am Acad Dermatol 2004;50:315-318.

-9 Takai T, Matsunaga A: A case of neutrophilic eccrine hidradenitis associated with streptococcal infectious endocarditis. Dermatology 2006;212:203-205. 
10 Landau M, Metzker A, Gat A, Ben-Amitai D, Brenner S: Palmoplantar eccrine hidradenitis: three new cases and review. Pediatr Dermatol 1998;15:97-102.

11 Lee WJ, Kim CH, Chang SE, Lee MW, Choi JH, Moon KC, Koh JK: Generalized idiopathic neutrophilic eccrine hidradenitis in childhood. Int J Dermatol 2010;49:75-78.

12 Shih IH, Huang YH, Yang CH, Yang LC, Hong HS: Childhood neutrophilic eccrine hidradenitis: a clinicopathologic and immunohistochemical study of 10 patients. J Am Acad Dermatol 2005;52:963-966.

13 Naimer SA, Zvulunov A, Ben-Amitai D, Landau M: Plantar hidradenitis in children induced by exposure to wet footwear. Pediatr Emerg Care 2000;16:182-183.

14 Wenzel FG, Horn TD: Nonneoplastic disorders of the eccrine glands. J Am Acad Dermatol 1998;38:1-17; quiz 18-20.

15 Rieg S, Garbe C, Sauer B, Kalbacher H, Schittek B: Dermcidin is constitutively produced by eccrine sweat glands and is not induced in epidermal cells under inflammatory skin conditions. Br J Dermatol 2004;151:534-539. 